\title{
STOCHASTIC COMPARISONS OF RESIDUAL LIFETIMES AND INACTIVITY TIMES OF COHERENT SYSTEMS
}

\author{
NITIN GUPTA, ${ }^{*}$ Jaypee University of Information Technology and \\ Indian Institute of Technology Kharagpur
}

\begin{abstract}
Under the assumption of independent and identically distributed (i.i.d.) components, the problem of the stochastic comparison of a coherent system having used components and a used coherent system has been considered. Necessary and sufficient conditions on structure functions have been provided for the stochastic comparison of a coherent system having used/inactive i.i.d. components and a used/inactive coherent system. As a consequence, for $r$-out-of- $n$ systems, it has been shown that systems having used i.i.d. components stochastically dominate used systems in the likelihood ratio ordering.
\end{abstract}

Keywords: Likelihood ratio order; coherent system; residual life; inactivity time

2010 Mathematics Subject Classification: Primary 90B25

Secondary 60E15

\section{Introduction}

In some practical situations, one has to make a choice between a used system of $n$ components and a system made up of $n$ used components. The used system or the system made up of used components has a lifetime in terms of the residual life. Let $X$ be a random variable with probability density function $f(\cdot)$, distribution function $F(\cdot)$, and survival function $\bar{F}(\cdot)=$ $1-F(\cdot)$. The residual lifetime and the inactivity time of $X$ with age/time $t \geq 0$ is defined as

$$
X_{t}=(X-t \mid X>t) \text { and } X_{(t)}=(t-X \mid X \leq t) \text {, }
$$

respectively. For comprehensive details on the residual lifetime and the inactivity time, we refer the reader to [2], [3], and [12]. The stochastic comparisons and reliability properties of the residual lifetime and the inactivity time have been discussed by [6], [7], [10], [11], and [14].

Let us denote by $\eta(\cdot)=-f^{\prime}(\cdot) / f(\cdot)$, the eta function of the random variable $X$. The eta function plays a vital role in the study of the reliability characteristics. We refer the reader to [4] for an overview of the eta function.

Throughout this paper, terms such as 'increasing' and 'decreasing' will be used to denote 'nondecreasing' and 'nonincreasing', respectively. To make the paper self-contained, we include below some definitions which are standard in the literature (see [13]).

Definition 1.1. Let $Z_{i}, i=1,2$, be two random variables with probability density functions $g_{i}(\cdot)$, distribution functions $G_{i}(\cdot)$, and survival functions $\bar{G}_{i}(\cdot)=1-G_{i}(\cdot), i=1,2$. Then the

Received 15 March 2012; revision received 12 December 2012.

* Postal address: Department of Mathematics, Indian Institute of Technology Kharagpur, Kharagpur-721302, India.

Email address: nitin.gupta@maths.iitkgp.ernet.in 
random variable $Z_{1}$ is said to be smaller than the random variable $Z_{2}$ in the

(a) likelihood ratio ordering (written as $\left.Z_{1} \leq_{\operatorname{lr}} Z_{2}\right)$ if $g_{2}(x) / g_{1}(x)$ increases in $x$ on $(0, \infty)$;

(b) failure rate ordering (written as $\left.Z_{1} \leq_{\mathrm{fr}} Z_{2}\right)$ if $\bar{G}_{2}(x) / \bar{G}_{1}(x)$ increases in $x$ on $(0, \infty)$;

(c) usual stochastic ordering (written as $Z_{1} \leq$ st $Z_{2}$ ) if $\bar{G}_{1}(x) \leq \bar{G}_{2}(x)$ for all $x \in \mathbb{R}$.

The following implications/equivalances are well known in the literature (see, for example, $[13$, p. 43]):

$$
\begin{gathered}
Z_{1} \leq_{\operatorname{lr}} Z_{2} \Rightarrow Z_{1} \leq_{\mathrm{fr}} Z_{2} \Rightarrow Z_{1} \leq_{\mathrm{st}} Z_{2}, \\
Z_{1} \leq_{\operatorname{lr}} Z_{2} \Longleftrightarrow \bar{G}_{2} \bar{G}_{1}^{-1}(p) \text { is concave in } p \text { on }(0,1) .
\end{gathered}
$$

An $r$-out-of- $n$ system functions if at least $r$ out of $n$ components of the system function, $r=\{1,2, \ldots, n\}$. This $r$-out-of- $n$ system is a particular case of a general coherent system. Series and parallel systems are two important reliability structures. The series system is an $n$-out-of- $n$ system and the parallel system is a 1-out-of- $n$ system. For details on general coherent structures, we refer the reader to [1].

We now provide a brief review of the results in the literature about reliability properties of the residual lifetime and the inactivity time. These results are related to the results proved in this paper. Zhang and Li [14] proved that the lifetime of a parallel/series system having independently and identically distributed (i.i.d.) components stochastically dominates the lifetime of a used parallel/series system in the usual stochastic ordering; they also derived similar results for the inactivity time. For i.i.d. components, $\mathrm{Li}$ and $\mathrm{Lu} \mathrm{[6]} \mathrm{strengthened} \mathrm{the} \mathrm{result}$ of Zhang and Li [14] from the usual stochastic ordering to the likelihood ratio ordering. For independent but not necessarily identically distributed components, $\mathrm{Li}$ and $\mathrm{Lu}$ [6] proved that the result of Zhang and $\mathrm{Li}$ [14] can be extended to the failure rate ordering. For independent but not necessarily identically distributed components, Gupta et al. [5] recently derived the conditions under which the results of $\mathrm{Li}$ and $\mathrm{Lu} \mathrm{[6]} \mathrm{can} \mathrm{be} \mathrm{extended} \mathrm{to} \mathrm{the} \mathrm{likelihood} \mathrm{ratio}$ ordering. For general coherent systems, Pellerey and Petakos [11] proved that the lifetime of a coherent system composed of used components dominates the lifetime of the used coherent system in the usual stochastic ordering.

In Section 2 we provide necessary and sufficient conditions on the structure functions so that the lifetime of a coherent system having used/inactive i.i.d. components perform better/worse than the lifetime of a used/inactive coherent system under the likelihood ratio ordering. Consequently, it follows that an $r$-out-of- $n$ system having used i.i.d. components stochastically dominates the used $r$-out-of- $n$ system under the likelihood ratio ordering. A few examples have been provided to illustrate the situations in which the main results of the paper may provide comparative analysis. Also, some results of $\mathrm{Li}$ and $\mathrm{Lu}$ [6] have been obtained as the particular case of the main results of the paper.

Intuitively, a system of used but functioning components is better than a used system where the states of the individual components are unknown. Therefore, it is natural to ask whether this intuition is valid for all the systems. In Example 2.4 we show that there exist such particular systems in which a used system is better than a system of used but functioning components in the likelihood ratio ordering.

\section{Stochastic comparisons}

Let us consider a coherent system, with structure function $\phi$, having $n$ components $C_{1}, \ldots$, $C_{n}$ with i.i.d. lifetimes as $X_{1}, \ldots, X_{n}$, respectively. Let the i.i.d. components have probability 
density function $f(\cdot)$, distribution function $F(\cdot)$, and survival function $\bar{F}(\cdot)=1-F(\cdot)$. The lifetime of coherent system $\phi$ is denoted by $\tau(\boldsymbol{X})=\tau\left(X_{1}, \cdots, X_{n}\right)$ with the survival function as

$$
\mathbb{P}(\tau(\boldsymbol{X})>x)=K(\bar{F}(x)), \quad x \in \mathbb{R},
$$

where $K:[0,1] \rightarrow[0,1]$ is an increasing function having $K(0)=0$ and $K(1)=1$.

The residual life of a coherent system having i.i.d. components $X_{1}, \ldots, X_{n}$ is

$$
(\tau(\boldsymbol{X}))_{t}=\left(\tau\left(X_{1}, \ldots, X_{n}\right)\right)_{t} .
$$

Then the resulting used coherent system, denoted by $S_{S}$, has survival function

$$
\bar{F}_{S}(x)=\mathbb{P}\left((\tau(\boldsymbol{X}))_{t}>x\right)=\frac{\mathbb{P}(\tau(\boldsymbol{X})>t+x)}{\mathbb{P}(\tau(\boldsymbol{X})>t)}= \begin{cases}1 & \text { if } x<0, \\ \frac{K(\bar{F}(t+x))}{K(\bar{F}(t))} & \text { if } x \geq 0 .\end{cases}
$$

The lifetime of a coherent system having residual lives $\left(X_{1}\right)_{t}, \ldots,\left(X_{n}\right)_{t}$ is

$$
\tau\left(\boldsymbol{X}_{\boldsymbol{t}}\right)=\tau\left(\left(X_{1}\right)_{t}, \ldots,\left(X_{n}\right)_{t}\right) .
$$

Then the resulting coherent system having used components, denoted by $S_{C}$, has survival function

$$
\bar{F}_{C}(x)=\mathbb{P}\left(\tau\left(\boldsymbol{X}_{\boldsymbol{t}}\right)>x\right)= \begin{cases}1 & \text { if } x<0 \\ K\left(\frac{\bar{F}(t+x)}{\bar{F}(t)}\right) & \text { if } x \geq 0 .\end{cases}
$$

Here we investigate the equivalent conditions for

$$
(\tau(\boldsymbol{X}))_{t} \leq_{\operatorname{lr}}\left(\geq_{\operatorname{lr}}\right) \tau\left(\boldsymbol{X}_{\boldsymbol{t}}\right) \text { for all } t \geq 0
$$

to hold. The following theorem provides the conditions under which a coherent system of used but functioning components performs better/worse than a used coherent system in the likelihood ratio ordering.

Theorem 2.1. It holds that $(\tau(\boldsymbol{X}))_{t} \leq_{\mathrm{lr}}\left(\geq_{\mathrm{lr}}\right) \tau\left(\boldsymbol{X}_{\boldsymbol{t}}\right)$ if and only if, for fixed $t \geq 0$ and $q \in(0,1)$, one of the following conditions hold:

(a) $K\left(q K^{-1}(p)\right)$ is a convex (concave) function of $p$ on $(0,1)$;

(b) $\psi_{1, q}(p)=K^{\prime}(p / q) / K^{\prime}(p)$ is a decreasing (increasing) function of $p$ on $(0,1), p<q$;

(c) $K^{\prime \prime}(p) / K^{\prime}(p) \geq(\leq) K^{\prime \prime}(p / q) / q K^{\prime}(p / q)$ for any $p$ on $(0,1), p<q$;

where $K^{\prime}(\cdot)$ and $K^{\prime \prime}(\cdot)$ denote the first and second derivatives of $K(\cdot)$, respectively.

Proof. Let $t \geq 0$ be fixed. The survival function, the probability density function, and the eta function of $(\tau(\boldsymbol{X}))_{t}$ are given by (2.1),

$$
f_{S}(x)=f(t+x) \frac{K^{\prime}(\bar{F}(t+x))}{K(\bar{F}(t))}, \quad x \geq 0, \quad \text { and } \quad \eta_{S}(x)=-\frac{f_{S}^{\prime}(x)}{f_{S}(x)}, \quad x \geq 0,
$$

respectively. Similarly, the survival function, the probability density function, and the eta function of $\tau\left(\boldsymbol{X}_{\boldsymbol{t}}\right)$ are given by (2.2),

$$
f_{C}(x)=\frac{f(t+x)}{\bar{F}(t)} K^{\prime}\left(\frac{\bar{F}(t+x)}{\bar{F}(t)}\right), \quad x \geq 0, \quad \text { and } \quad \eta_{C}(x)=-\frac{f_{C}^{\prime}(x)}{f_{C}(x)}, \quad x \geq 0,
$$

respectively. 
(a) Now $(\tau(\boldsymbol{X}))_{t} \leq \mathrm{lr}\left(\geq_{\mathrm{lr}}\right) \tau\left(\boldsymbol{X}_{\boldsymbol{t}}\right)$ if and only if $\bar{F}_{S} \bar{F}_{C}^{-1}(p)$ is a convex (concave) function of $p$ on $(0,1)$. Also, for any $p \in(0,1)$,

$$
\bar{F}_{S} \bar{F}_{C}^{-1}(p)=\bar{F}_{S}\left(\bar{F}^{-1}\left(\bar{F}(t) K^{-1}(p)\right)-t\right)=\frac{\left.K(\bar{F}(t)) K^{-1}(p)\right)}{K(\bar{F}(t))} .
$$

Therefore, $(\tau(\boldsymbol{X}))_{t} \leq \operatorname{lr}\left(\geq_{\operatorname{lr}}\right) \tau\left(\boldsymbol{X}_{t}\right)$ if and only if $K\left(q K^{-1}(p)\right)$ is a convex (concave) function of $p$ on $(0,1)$ for fixed $q \in(0,1)$.

(b) Now $(\tau(\boldsymbol{X}))_{t} \leq_{\operatorname{lr}}\left(\geq_{\operatorname{lr}}\right) \tau\left(\boldsymbol{X}_{\boldsymbol{t}}\right)$ if and only if

$$
m_{1, t}(x)=\frac{f_{C}(x)}{f_{S}(x)}=\frac{K(\bar{F}(t))}{\bar{F}(t)} \frac{K^{\prime}(\bar{F}(t+x) / \bar{F}(t))}{K^{\prime}(\bar{F}(t+x))}=A(t) m_{2, t}(x)
$$

is an increasing (decreasing) function of $x$ on $(0, \infty)$, where

$$
A(t)=\frac{K(\bar{F}(t))}{\bar{F}(t)} \quad \text { and } \quad m_{2, t}(x)=\frac{K^{\prime}(\bar{F}(t+x) / \bar{F}(t))}{K^{\prime}(\bar{F}(t+x))} .
$$

Equivalently, $(\tau(\boldsymbol{X}))_{t} \leq_{\operatorname{lr}}\left(\geq_{\mathrm{lr}}\right) \tau\left(\boldsymbol{X}_{\boldsymbol{t}}\right)$ if and only if $\psi_{1, q}(p)=K^{\prime}(p / q) / K^{\prime}(p)$ is a decreasing (increasing) function of $p$ on $(0,1)$ for any $q \in(0,1), p<q$.

(c) Note that

$$
\begin{aligned}
(\tau(\boldsymbol{X}))_{t} \leq \operatorname{lr}(\geq \operatorname{lr}) \tau\left(\boldsymbol{X}_{t}\right) & \Longleftrightarrow \ln \left(\frac{f_{C}(x)}{f_{S}(x)}\right) \text { is increasing (decreasing) in } x \in(0, \infty) \\
& \Longleftrightarrow \eta_{S}(x) \geq(\leq) \eta_{C}(x) \text { for all } x \in(0, \infty) .
\end{aligned}
$$

Consider

$$
\begin{aligned}
\eta_{S}(x)-\eta_{C}(x)= & -\frac{f_{S}^{\prime}(x)}{f_{S}(x)}+\frac{f_{C}^{\prime}(x)}{f_{C}(x)} \\
= & -\frac{f^{\prime}(t+x) K^{\prime}(\bar{F}(t+x))-f^{2}(t+x) K^{\prime \prime}(\bar{F}(t+x))}{f(t+x) K^{\prime}(\bar{F}(t+x))} \\
& +\frac{f^{\prime}(t+x) K^{\prime}(\bar{F}(t+x) / \bar{F}(t))-f^{2}(t+x) K^{\prime \prime}(\bar{F}(t+x) / \bar{F}(t)) / \bar{F}(t)}{f(t+x) K^{\prime}(\bar{F}(t+x) / \bar{F}(t))} \\
= & \frac{f(t+x) K^{\prime \prime}(\bar{F}(t+x))}{K^{\prime}(\bar{F}(t+x))}-\frac{f(t+x) K^{\prime \prime}(\bar{F}(t+x) / \bar{F}(t))}{\bar{F}(t) K^{\prime}(\bar{F}(t+x) / \bar{F}(t))} \\
= & f(t+x)\left(\frac{K^{\prime \prime}(\bar{F}(t+x))}{K^{\prime}(\bar{F}(t+x))}-\frac{K^{\prime \prime}(\bar{F}(t+x) / \bar{F}(t))}{\bar{F}(t) K^{\prime}(\bar{F}(t+x) / \bar{F}(t))}\right) .
\end{aligned}
$$

Therefore, it is clear that

$$
(\tau(\boldsymbol{X}))_{t} \leq \operatorname{lr}\left(\geq_{\operatorname{lr}}\right) \tau\left(\boldsymbol{X}_{\boldsymbol{t}}\right)
$$

if and only if

$$
\frac{K^{\prime \prime}(p)}{K^{\prime}(p)} \geq(\leq) \frac{K^{\prime \prime}(p / q)}{q K^{\prime}(p / q)}
$$

for any $p, q$ on $(0,1), p<q$. 
Corollary 2.1. Let $r \in\{1,2, \ldots, n\}$. Then, for $r$-out-of-n systems,

$$
(\tau(\boldsymbol{X}))_{t} \leq \operatorname{lr} \tau\left(\boldsymbol{X}_{\boldsymbol{t}}\right) .
$$

Proof. For $r$-out-of- $n$ systems (see [9]),

$$
K(p)=\frac{n !}{(n-r) !(r-1) !} \int_{1-p}^{1} u^{n-r}(1-u)^{r-1} \mathrm{~d} u, \quad p \in(0,1),
$$

and

$$
K^{\prime}(p)=\frac{n !}{(n-r) !(r-1) !}(1-p)^{n-r} p^{r-1} .
$$

Therefore, for fixed $q \in(0,1)$,

$$
\psi_{1, q}(p)=\frac{K^{\prime}(p / q)}{K^{\prime}(p)}=\frac{1}{q^{n-1}}\left(\frac{q-p}{1-p}\right)^{n-r}=\frac{1}{q^{n-1}}\left(1-\frac{1-q}{1-p}\right)^{n-r}
$$

is a decreasing function of $p$ on $(0,1)$. The result follows from Theorem 2.1.

Remark 2.1. (a) The referee observed that the conditions provided in Theorem 2.1 depend only on the structure of the system, not on the component lifetime distribution, provided that the density is everywhere positive. Moreover, Corollary 2.1 shows that an $r$-out-of- $n$ system of used but functioning components is better than a used $r$-out-of- $n$ system where the states of the individual components are unknown.

(b) For a parallel system (i.e. 1-out-of- $n$ system),

$$
\tau(\boldsymbol{X})=\max \left(X_{1}, \ldots, X_{n}\right) .
$$

Then, from Corollary 2.1, it is clear that

$$
\left(\max \left(X_{1}, \ldots, X_{n}\right)\right)_{t} \leq_{\operatorname{lr}} \max \left(\left(X_{1}\right)_{t}, \ldots,\left(X_{n}\right)_{t}\right) .
$$

The result stated in Remark 2.1(b) was proved by Li and Lu [6].

The following examples illustrate the situations in which Theorem 2.1 may provide a comparative analysis between a system of used but functioning i.i.d. components and a used system having i.i.d. components.

Example 2.1. Consider the example of a relay circuit (see Figure 1.3.4 of [1, p. 13]) with i.i.d. components and lifetime

$$
\tau(\boldsymbol{X})=\min \left(X_{1}, \max \left(X_{2}, X_{3}\right)\right) \text {. }
$$

Then, for this system,

$$
K(p)=2 p^{2}-p^{3}
$$

and

$$
K^{\prime}(p)=4 p-3 p^{2}
$$

Hence, for fixed $q \in(0,1)$,

$$
\psi_{1, q}(p)=\frac{K^{\prime}(p / q)}{K^{\prime}(p)}=\frac{1}{q^{2}} \frac{4 q-3 p}{4-3 p}=\frac{1}{q^{2}}\left(1+\frac{q-1}{4-3 p}\right)
$$

is a decreasing function of $p$ on $(0,1)$. Therefore, using Theorem 2.1 , it follows that a relay circuit of used but functioning components is better then a used relay circuit in the likelihood ratio order. 
Example 2.2. Suppose that three i.i.d. components are connected in parallel, and then two such parallel connections are hooked up in series to form a single system (see Exercise 11.16 with $k=3$ and $n=2$ of [8]) with lifetime

$$
\tau(\boldsymbol{X})=\min \left(\max \left(X_{1}, X_{2}, X_{3}\right), \max \left(X_{4}, X_{5}, X_{6}\right)\right) .
$$

Then, for such a system,

$$
K(p)=\left(1-(1-p)^{3}\right)^{2}=\left(p^{3}-3 p^{2}+3 p\right)^{2}
$$

and

$$
K^{\prime}(p)=6 p\left(p^{2}-3 p+3\right)(p-1)^{2} .
$$

Hence, for fixed $q \in(0,1)$,

$$
\psi_{1, q}(p)=\frac{K^{\prime}(p / q)}{K^{\prime}(p)}=\frac{1}{q^{5}} \eta_{1, q}(p) \eta_{2, q}(p),
$$

where

$$
\eta_{1, q}(p)=\frac{p^{2}-3 p q+3 q^{2}}{p^{2}-3 p+3}
$$

and

$$
\eta_{2, q}(p)=\left(\frac{p-q}{p-1}\right)^{2} \text {. }
$$

Consider, for $p \leq q$,

$$
\begin{aligned}
\eta_{1, q}^{\prime}(p) & =\frac{1}{\left(p^{2}-3 p+3\right)^{2}}\left(\left(p^{2}-3 p+3\right)(2 p-3 q)-\left(p^{2}-3 p q+3 q^{2}\right)(2 p-3)\right) \\
& =\frac{1}{\left(p^{2}-3 p+3\right)^{2}}\left(-3 p^{2} q+3 p^{2}-6 p^{2}+6 p^{2} q+6 p-6 p q^{2}-9 q+9 q^{2}\right) \\
& =\frac{1}{\left(p^{2}-3 p+3\right)^{2}}\left(-9 p^{2}(1-q)+6 p\left(1-q^{2}\right)-9 q(1-q)\right) \\
& =\frac{1}{\left(p^{2}-3 p+3\right)^{2}} 3(1-q)\left(-3 p^{2}+2 p(1+q)-9 q\right) \\
& =\frac{1}{\left(p^{2}-3 p+3\right)^{2}} 3(1-q)\left(-3 p^{2}+2(p-q)-4 q-2 q(1-p)\right) \\
& \leq 0
\end{aligned}
$$

therefore, $\eta_{1, q}(p)$ is a decreasing function of $p$ on $(0,1), p \leq q$. Also, it is easy to see that $\eta_{2, q}(p)$ is a decreasing function of $p$ on $(0,1), p \leq q$. Since $\eta_{1, q}(p) \geq 0, \eta_{2, q}(p) \geq 0$, and both are decreasing functions of $p$ on $(0,1), p \leq q$, from $(2.3)$, it follows that $\psi_{1, q}(p)$ is a decreasing function of $p$ on $(0,1), p \leq q$. Now, for the considered system of this example, using Theorem 2.1, it follows that

$$
(\tau(\boldsymbol{X}))_{t} \leq \operatorname{lr} \tau\left(\boldsymbol{X}_{\boldsymbol{t}}\right) .
$$

Example 2.3. Consider the example of a stereo hi-fi system (see Example 1.5 of $[1$, p. 4]) with i.i.d. components and lifetime

$$
\tau(\boldsymbol{X})=\min \left(\max \left(X_{1}, X_{2}\right), X_{3}, \max \left(X_{4}, X_{5}\right)\right) .
$$

Then, for this system,

$$
K(p)=4 p^{3}-4 p^{4}+p^{5} \quad \text { and } \quad K^{\prime}(p)=12 p^{2}-16 p^{3}+5 p^{4} .
$$


Hence, for fixed $q \in(0,1)$,

$$
\begin{aligned}
\psi_{1, q}(p) & =\frac{K^{\prime}(p / q)}{K^{\prime}(p)} \\
& =\frac{1}{q^{4}} \frac{12 q^{2}-16 p q+5 p^{2}}{12-16 p+5 p^{2}} \\
& =\frac{1}{q^{4}}\left(1+\frac{12\left(q^{2}-1\right)+16 p(1-q)}{12-16 p+5 p^{2}}\right) \\
& =\frac{1}{q^{4}}\left(1+(1-q) \eta_{3, q}(p)\right),
\end{aligned}
$$

where

$$
\eta_{3, q}(p)=\frac{16 p-12(q+1)}{12-16 p+5 p^{2}}
$$

Also, for $p \leq q$,

$$
\begin{aligned}
\eta_{3, q}^{\prime}(p) & =\frac{1}{\left(12-16 p+5 p^{2}\right)^{2}}\left(16\left(12-16 p+5 p^{2}\right)-(16 p-12(q+1))(-16+10 p)\right) \\
& =\frac{1}{\left(12-16 p+5 p^{2}\right)^{2}}\left(10 p(12-16 p)+80 p^{2}+12 q(-16+10 p)\right) \\
& =\frac{1}{\left(12-16 p+5 p^{2}\right)^{2}}\left(120 p-80 p^{2}-292 q+120 p q\right) \\
& \leq \frac{1}{\left(12-16 p+5 p^{2}\right)^{2}}\left(120 q-80 p^{2}-292 q+120 p q\right) \\
& =\frac{1}{\left(12-16 p+5 p^{2}\right)^{2}}\left(120 q(1-p)-80 p^{2}-292 q\right) \\
& \leq \frac{1}{\left(12-16 p+5 p^{2}\right)^{2}}\left(120 q-80 p^{2}-292 q\right) \\
& \leq 0
\end{aligned}
$$

therefore, $\eta_{3, q}(p)$ is decreasing function of $p$ on $(0,1), p \leq q$. Hence, from (2.4), it is clear that $\psi_{1, q}(p)$ is a decreasing function of $p$ on $(0,1), p \leq q$. Now, using Theorem 2.1, it follows that a stereo hi-fi system of used but functioning components is better then a used stereo hi-fi system in the likelihood ratio order.

Since, intuitively, a system of used but functioning components is better than a used system where the states of the individual components are unknown, the referee raised the natural question of whether the relation $(\tau(\boldsymbol{X}))_{t} \leq_{\operatorname{lr}} \tau\left(\boldsymbol{X}_{\boldsymbol{t}}\right)$ is valid for all systems. The following example shows that there exist such systems for which $(\tau(\boldsymbol{X}))_{t} \geq_{\operatorname{lr}} \tau\left(\boldsymbol{X}_{\boldsymbol{t}}\right)$ holds.

Example 2.4. Consider the lifetime of coherent system to be

$$
\tau(\boldsymbol{X})=X_{1}+X_{2},
$$

where $X_{1}$ and $X_{2}$ follow exponential distributions with mean $1 / \lambda$. Then the survival function and the probability density function of a used coherent system are

$$
\bar{F}_{S}(x)=\mathbb{P}\left(\left(X_{1}+X_{2}\right)_{t}>x\right)=\frac{1}{\mathbb{P}\left(X_{1}+X_{2}>t\right)} \int \bar{F}(x+t-z) f(z) \mathrm{d} z
$$


and

$$
\begin{aligned}
f_{S}(x) & =\frac{1}{\mathbb{P}\left(X_{1}+X_{2}>t\right)} \int f(x+t-z) f(z) \mathrm{d} z \\
& =\frac{1}{\mathbb{P}\left(X_{1}+X_{2}>t\right)} \int_{0}^{x+t} \lambda^{2} \mathrm{e}^{-\lambda(x+t-z)} \mathrm{e}^{-\lambda z} \mathrm{~d} z \\
& =\frac{1}{\mathbb{P}\left(X_{1}+X_{2}>t\right)} \lambda^{2}(x+t) \mathrm{e}^{-\lambda(x+t)},
\end{aligned}
$$

respectively. Also, the survival function and the probability density function of a coherent system with used components are

$$
\bar{F}_{C}(x)=\mathbb{P}\left(\left(X_{1}\right)_{t}+\left(X_{2}\right)_{t}>x\right)=\frac{1}{\bar{F}^{2}(t)} \int \bar{F}(x+t-z) f(z+t) \mathrm{d} z
$$

and

$$
\begin{aligned}
f_{C}(x) & =\frac{1}{\bar{F}^{2}(t)} \int f(x+t-z) f(z+t) \mathrm{d} z \\
& =\frac{1}{\mathrm{e}^{-2 \lambda t}} \int_{-t}^{x+t} \lambda^{2} \mathrm{e}^{-\lambda(x+t-z)} \mathrm{e}^{-\lambda(t+z)} \mathrm{d} z \\
& =\lambda^{2}(x+2 t) \mathrm{e}^{-\lambda x},
\end{aligned}
$$

respectively. Consider

$$
\frac{f_{C}(x)}{f_{S}(x)}=\frac{\mathbb{P}\left(X_{1}+X_{2}>t\right)}{\mathrm{e}^{-\lambda t}} \frac{x+2 t}{x+t}=\frac{\mathbb{P}\left(X_{1}+X_{2}>t\right)}{\mathrm{e}^{-\lambda t}}\left(1+\frac{t}{x+t}\right),
$$

which is a decreasing function of $x$ on $(0, \infty)$. Hence,

$$
\left(X_{1}+X_{2}\right)_{t} \geq_{\operatorname{lr}}\left(X_{1}\right)_{t}+\left(X_{2}\right)_{t},
$$

where $X_{1}$ and $X_{2}$ follow exponential distributions with mean $1 / \lambda$.

Remark 2.2. Pellerey and Petakos [11] in the proof of their Theorem 1 claimed that

$$
(\tau(\boldsymbol{X}))_{t} \leq_{\mathrm{st}} \tau\left(\boldsymbol{X}_{\boldsymbol{t}}\right) \text { for all } t \geq 0
$$

(see Theorem 1, Equation (7) of [11]). They assumed in their Equation (4) that

$$
\left\{X_{i}>s \text { for all } i \in I\right\} \subseteq\{\tau(\boldsymbol{X})>s\} \text { for all } s>0 .
$$

Therefore, (2.5) is restricted to coherent systems where relation (2.6) holds. But, there may exist coherent structures where relation (2.6) may not be satisfied, e.g. $\tau(\boldsymbol{X})=X_{1}+X_{2}$. From implication (1.1) and Example 2.4, it follows that if $\tau(\boldsymbol{X})=X_{1}+X_{2}$, where $X_{1}$ and $X_{2}$ follow exponential distributions with mean $1 / \lambda$, then

$$
\left(X_{1}+X_{2}\right)_{t} \geq_{\mathrm{st}}\left(X_{1}\right)_{t}+\left(X_{2}\right)_{t}
$$

Therefore, if condition (2.6) does not hold for a coherent structure then the relation

$$
(\tau(\boldsymbol{X}))_{t} \leq_{\mathrm{st}} \tau\left(\boldsymbol{X}_{\boldsymbol{t}}\right) \text { for all } t \geq 0
$$

may or may not hold. 
In the remainder of this paper, we compare a coherent system of inactive components and an inactive coherent system in the likelihood ratio ordering. The inactivity time of a coherent system with i.i.d. components $X_{1}, \ldots, X_{n}$ is

$$
(\tau(\boldsymbol{X}))_{(t)}=\left(\tau\left(X_{1}, \ldots, X_{n}\right)\right)_{(t)} .
$$

Then the resulting inactive coherent system with inactivity time $t \geq 0$, denoted by $S_{I, S}$, has survival function

$$
\bar{F}_{I, S}(x)=\mathbb{P}\left((\tau(\boldsymbol{X}))_{(t)}>x\right)= \begin{cases}1 & \text { if } x<0, \\ \frac{1-K(\bar{F}(t-x))}{1-K(\bar{F}(t))} & \text { if } 0 \leq x<t, \\ 0 & \text { if } x \geq t .\end{cases}
$$

The lifetime of a coherent system with inactivity times $\left(X_{1}\right)_{(t)}, \ldots,\left(X_{n}\right)_{(t)}$ is

$$
\tau\left(\boldsymbol{X}_{(t)}\right)=\tau\left(\left(X_{1}\right)_{(t)}, \ldots,\left(X_{n}\right)_{(t)}\right) .
$$

Then the resulting coherent system with inactive components, denoted by $S_{I, C}$, has survival function

$$
\bar{F}_{I, C}(x)=\mathbb{P}\left(\tau\left(\boldsymbol{X}_{(t)}\right)>x\right)= \begin{cases}1 & \text { if } x<0, \\ K\left(\frac{1-\bar{F}(t-x)}{1-\bar{F}(t)}\right) & \text { if } 0 \leq x<t \\ 0 & \text { if } x \geq t\end{cases}
$$

Here we investigate the equivalent conditions for

$$
(\tau(\boldsymbol{X}))_{(t)} \leq \operatorname{lr}\left(\geq_{\operatorname{lr}}\right) \tau\left(\boldsymbol{X}_{(t)}\right) \text { for all } t \geq 0
$$

to hold. The following theorem provides the conditions under which a coherent system of inactive components performs better/worse than an inactive coherent system in likelihood ratio ordering.

Theorem 2.2. It holds that $(\tau(\boldsymbol{X}))_{(t)} \leq_{\operatorname{lr}}\left(\geq_{\mathrm{lr}}\right) \tau\left(\boldsymbol{X}_{(t)}\right)$ if and only if, for fixed $t \geq 0$ and $q \in(0,1)$, one of the following conditions hold:

(a) $1-K\left(1-q K^{-1}(p)\right)$ is a convex (concave) function of $p$ on $(0,1)$;

(b) $\psi_{2, q}(p)=K^{\prime}((1-p) /(1-q)) / K^{\prime}(p)$ is a increasing (decreasing) function of $p$ on $(0,1), p>q$;

(c) $K^{\prime \prime}(p) / K^{\prime}(p)+K^{\prime \prime}((1-p) /(1-q)) /(1-q) K^{\prime}((1-p) /(1-q)) \leq(\geq) 0$ for any $p$ on $(0,1), p>q$;

where $K^{\prime}(\cdot)$ and $K^{\prime \prime}(\cdot)$ denote the first and second derivatives of $K(\cdot)$, respectively.

Proof. Let $t \geq 0$ be fixed. The survival function, the probability density function, and the eta function of $(\tau(\boldsymbol{X}))_{(t)}$ are respectively given by (2.7),

$$
\begin{array}{cl}
f_{I, S}(x)=f(t-x) \frac{K^{\prime}(\bar{F}(t-x))}{1-K(\bar{F}(t))}, & t>x \geq 0, \\
\text { and } \quad \eta_{I, S}(x)=-\frac{f_{I, S}^{\prime}(x)}{f_{I, S}(x)}, \quad x \geq 0 .
\end{array}
$$


Similarly, the survival function, the probability density function, and the eta function of $\tau\left(\boldsymbol{X}_{(t)}\right)$ are respectively given by (2.8),

$$
\begin{gathered}
f_{I, C}(x)=\frac{f(t-x)}{1-\bar{F}(t)} K^{\prime}\left(\frac{1-\bar{F}(t-x)}{1-\bar{F}(t)}\right), \quad t>x \geq 0, \\
\text { and } \quad \eta_{I, C}(x)=-\frac{f_{I, C}^{\prime}(x)}{f_{I, C}(x)}, \quad x \geq 0 .
\end{gathered}
$$

(a) Now $(\tau(\boldsymbol{X}))_{t} \leq_{\operatorname{lr}}\left(\geq_{\operatorname{lr}}\right) \tau\left(\boldsymbol{X}_{\boldsymbol{t}}\right)$ if and only if $\bar{F}_{I, S} \bar{F}_{I, C}^{-1}(p)$ is a convex (concave) function of $p$ on $(0,1)$. Also, for any $p \in(0,1)$,

$$
\begin{aligned}
\bar{F}_{I, S} \bar{F}_{I, C}^{-1}(p) & =\bar{F}_{I, S}\left(t-F^{-1}\left(F(t) K^{-1}(p)\right)\right) \\
& =\frac{1-K\left(\bar{F}\left(F^{-1}\left(F(t) K^{-1}(p)\right)\right)\right)}{1-K(\bar{F}(t))} \\
& =\frac{1-K\left(1-F(t) K^{-1}(p)\right)}{1-K(\bar{F}(t))} .
\end{aligned}
$$

Therefore, $(\tau(\boldsymbol{X}))_{t} \leq_{\operatorname{lr}}\left(\geq_{\operatorname{lr}}\right) \tau\left(\boldsymbol{X}_{\boldsymbol{t}}\right)$ if and only if $1-K\left(1-q K^{-1}(p)\right)$ is a convex (concave) function of $p$ on $(0,1)$ for fixed $q \in(0,1)$.

(b) Now $(\tau(\boldsymbol{X}))_{(t)} \leq_{\text {lr }}\left(\geq_{\text {lr }}\right) \tau\left(\boldsymbol{X}_{(\boldsymbol{t})}\right)$ if and only if

$$
m_{2, t}(x)=\frac{f_{I, C}(x)}{f_{I, S}(x)}=\frac{1-K(\bar{F}(t))}{1-\bar{F}(t)} \frac{K^{\prime}((1-\bar{F}(t-x)) /(1-\bar{F}(t)))}{K^{\prime}(\bar{F}(t-x))}=B(t) m_{3, t}(x)
$$

is an increasing (increasing) function of $x$ on $(0, \infty)$, where

$$
B(t)=\frac{1-K(\bar{F}(t))}{1-\bar{F}(t)} \quad \text { and } \quad m_{3, t}(x)=\frac{K^{\prime}((1-\bar{F}(t-x)) /(1-\bar{F}(t)))}{K^{\prime}(\bar{F}(t-x))} .
$$

Equivalently, $(\tau(\boldsymbol{X}))_{(t)} \leq_{\mathrm{lr}}\left(\geq_{\mathrm{lr}}\right) \tau\left(\boldsymbol{X}_{(t)}\right)$ if and only if $\psi_{2, q}(p)=K^{\prime}((1-p) /(1-q)) / K^{\prime}(p)$ is an increasing (decreasing) function of $p$ on $(0,1)$ for any $q \in(0,1), p>q$.

(c) Note that

$$
\begin{aligned}
(\tau(\boldsymbol{X}))_{(t)} \leq_{\mathrm{lr}}\left(\geq_{\mathrm{lr}}\right) \tau\left(\boldsymbol{X}_{(t)}\right) & \Longleftrightarrow \ln \left(\frac{f_{I, C}(x)}{f_{I, S}(x)}\right) \text { is increasing (decreasing) in } x \in(0, \infty) \\
& \Longleftrightarrow \eta_{I, S}(x) \geq(\leq) \eta_{I, C}(x) \quad \text { for all } x \in(0, \infty) .
\end{aligned}
$$

Consider

$$
\begin{aligned}
\eta_{I, S}(x)- & \eta_{I, C}(x) \\
= & -\frac{f_{I, S}^{\prime}(x)}{f_{I, S}(x)}+\frac{f_{I, C}^{\prime}(x)}{f_{I, C}(x)} \\
= & -\frac{-f^{\prime}(t-x) K^{\prime}(\bar{F}(t-x))+f^{2}(t-x) K^{\prime \prime}(\bar{F}(t-x))}{f(t-x) K^{\prime}(\bar{F}(t-x))} \\
& +\left(-f^{\prime}(t-x) K^{\prime}\left(\frac{1-\bar{F}(t-x)}{1-\bar{F}(t)}\right)-\frac{f^{2}(t-x) K^{\prime \prime}((1-\bar{F}(t-x)) /(1-\bar{F}(t)))}{1-\bar{F}(t)}\right) \\
& \quad \times\left(f(t-x) K^{\prime}\left(\frac{1-\bar{F}(t-x)}{1-\bar{F}(t)}\right)\right)^{-1}
\end{aligned}
$$




$$
\begin{aligned}
& =-\frac{f(t-x) K^{\prime \prime}(\bar{F}(t-x))}{K^{\prime}(\bar{F}(t+x))}-\frac{f(t-x) K^{\prime \prime}((1-\bar{F}(t-x)) /(1-\bar{F}(t)))}{(1-\bar{F}(t)) K^{\prime}((1-\bar{F}(t-x)) /(1-\bar{F}(t)))} \\
& =-f(t-x)\left(\frac{K^{\prime \prime}(\bar{F}(t-x))}{K^{\prime}(\bar{F}(t-x))}+\frac{K^{\prime \prime}((1-\bar{F}(t-x)) /(1-\bar{F}(t)))}{(1-\bar{F}(t)) K^{\prime}((1-\bar{F}(t-x)) /(1-\bar{F}(t)))}\right) .
\end{aligned}
$$

Therefore, it is clear that

$$
\left.(\tau(\boldsymbol{X}))_{(t)} \leq_{\operatorname{lr}}\left(\geq_{\operatorname{lr}}\right) \tau\left(\boldsymbol{X}_{(t)}\right)\right)
$$

if and only if

$$
\frac{K^{\prime \prime}(p)}{K^{\prime}(p)}+\frac{K^{\prime \prime}((1-p) /(1-q))}{(1-q) K^{\prime}((1-p) /(1-q))} \leq(\geq) 0
$$

for any $p, q$ on $(0,1), p>q$.

Remark 2.3. (a) The conditions provided in Theorem 2.2 depend only on the structure of the system, not on the component lifetime distribution, provided that the density is everywhere positive.

(b) Let $r \in\{1,2, \ldots, n\}$. Let $p>q, p, q \in(0,1)$. Then, for $r$-out-of- $n$ systems,

$$
\begin{aligned}
\psi_{2, q}(p) & =\frac{K^{\prime}((1-p) /(1-q))}{K^{\prime}(p)} \\
& =\frac{(1-(1-p) /(1-q))^{n-r}((1-p) /(1-q))^{r-1}}{p^{r-1}(1-p)^{n-r}} \\
& =\frac{(p-q)^{n-r}}{p^{r-1}(1-p)^{n-2 r+1}(1-q)^{r-1}}
\end{aligned}
$$

Consider the following cases.

Case I. For a 1-out-of- $n$ system, i.e. for a parallel system, from (2.9),

$$
\psi_{2, q}(p)=\left(\frac{p-q}{1-p}\right)^{n-1}
$$

which is an increasing function of $p \in(0,1)$ for any $q \in(0,1)$ and $p>q$. Hence,

$$
\left(\max \left(X_{1}, \ldots, X_{n}\right)\right)_{(t)} \leq_{\operatorname{lr}} \max \left(\left(X_{1}\right)_{(t)}, \ldots,\left(X_{n}\right)_{(t)}\right) .
$$

This result was proved by $\mathrm{Li}$ and $\mathrm{Lu}[6]$.

Case II. For an $n$-out-of- $n$ system, i.e. for a series system, from (2.9),

$$
\psi_{2, q}(p)=\frac{1}{(1-q)^{n-1}}\left(\frac{1-p}{p}\right)^{n-1},
$$

which is a decreasing function of $p \in(0,1)$ for any $q \in(0,1)$ and $p>q$. Hence,

$$
\left(\min \left(X_{1}, \ldots, X_{n}\right)\right)_{(t)} \geq_{\operatorname{lr}} \min \left(\left(X_{1}\right)_{(t)}, \ldots,\left(X_{n}\right)_{(t)}\right) .
$$

This result was proved by $\mathrm{Li}$ and $\mathrm{Lu}[6]$. 


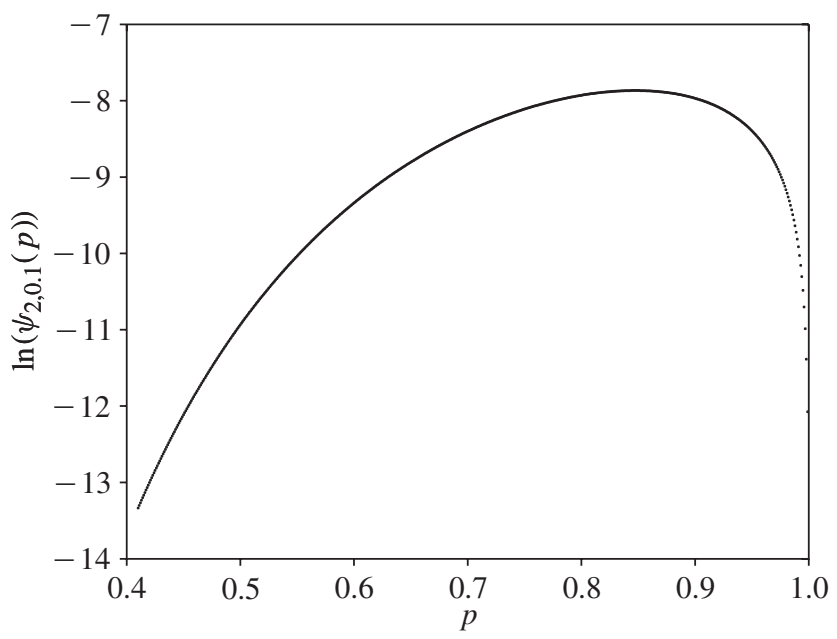

Figure 1: Plot of $\ln \left(\psi_{2,0.1}(p)\right)$ against $p$ for the 51-out-of-100 system.

Case III. For a 2-out-of-3 system, from (2.9),

$$
\psi_{2, q}(p)=\frac{1}{1-q}\left(1-\frac{q}{p}\right),
$$

which is an increasing function of $p \in(0,1)$ for any $q \in(0,1)$ and $p>q$. Hence, for a 2-out-of-3 system,

$$
(\tau(\boldsymbol{X}))_{(t)} \leq_{\operatorname{lr}} \tau\left(\boldsymbol{X}_{(\boldsymbol{t})}\right) .
$$

(c) From Figure 1, for a 51-out-of-100 system, it is clear that $\psi_{2,0.1}(p)$ is nonmonotonic in $p \in(0,1)$ for $p>q>0$. Hence, in general, the conditions of Theorem 2.2 may not be satisfied by an $r$-out-of- $n$ system, $r \in\{2,3, \ldots, n-1\}, r \neq 1, n$.

\section{Acknowledgements}

The author is grateful to the anonymous referee for his/her valuable comments and suggestions that have led to improvements in the article. The author acknowledges Prof. Harinder Singh, JUIT Waknaghat, for useful discussions related to Example 2.4 and Remark 2.2. The author also acknowledges Prof. Neeraj Misra, IIT Kanpur, for being a source of inspiration.

\section{References}

[1] Barlow, R. E. and Proschan, F. (1981). Statistical Theory of Reliability and Life Testing. Silver Spring, MD.

[2] Block, H. W., Savits, T. H. and Singh, H. (1998). The reversed hazard rate function. Prob. Eng. Inf. Sci. 12, 69-90.

[3] Chandra, N. K. and Roy, D. (2001). Some results on reversed hazard rate. Prob. Eng. Inf. Sci. 15, $95-102$.

[4] Glaser, R. E. (2001). Bathtub and related failure rate characterizations. J. Amer. Statist. Assoc. 75, 667-672.

[5] Gupta, N., Gandotra, N. and BajaJ, R. (2012). Reliability properties of residual life time and inactvity time of series and parallel system. J. Appl. Math. Statist. Inf. 8, 5-16.

[6] Li, X. AND Lu, J. (2003). Stochastic comparisons on residual life and inactivity time of series and parallel systems. Prob. Eng. Inf. Sci. 17, 267-275.

[7] Li, X. ANd Zuo, M. J. (2004). Stochastic comparison of residual life and inactivity time at a random time. Stoch. Models 20, 229-235.

[8] Meyer, P. L. (1970). Introductory Probability and Statistical Applications, 2nd edn. Addison-Wesley, Reading, MA. 
[9] Misra, N., Dhariyal, I. D. And Gupta, N. (2009). Optimal allocation of active spares in series systems and comparision of component and system redundancies. J. Appl. Prob. 46, 19-34.

[10] Misra, N., Gupta, N. And Dhariyal, I. D. (2008). Stochastic properties of residual life and inactivity time at a random time. Stoch. Models 24, 89-102.

[11] Pellerey, F. and Petakos, K. I. (2002). Closure property of the NBUC class under formation of parallel systems. IEEE Trans. Reliab. 51, 452-454.

[12] Ross, S. M. (1996). Stochastic Processes, 2nd edn. John Wiley, New York.

[13] Shaked, M. and Shanthikumar, J. G. (2007). Stochastic Orders. Springer, New York.

[14] Zhang, S. H. AND LI, X. H. (2003). Comparision between a system of used components and a used system. J. Lanzhou Univ. 39, 11-13. 